\title{
TTR
}

Traduction, terminologie, re?daction

\section{Lieven D'Hulst. Cent ans de théorie française de la traduction. De Batteux à Littré (1748-1847). Presses Universitaires de Lille, 1990, 256 p. ISBN 2-85939-370-6}

\section{Jean Delisle}

Volume 5, numéro 1, 1er semestre 1992

La pédagogie de la traduction : questions actuelles (1) et Miscellanées traductologiques

URI : https://id.erudit.org/iderudit/037116ar

DOI : https://doi.org/10.7202/037116ar

Aller au sommaire du numéro

Éditeur(s)

Association canadienne de traductologie

ISSN

0835-8443 (imprimé)

1708-2188 (numérique)

Découvrir la revue

Citer ce compte rendu

Delisle, J. (1992). Compte rendu de [Lieven D'Hulst. Cent ans de théorie française de la traduction. De Batteux à Littré (1748-1847). Presses Universitaires de Lille, 1990, 256 p. ISBN 2-85939-370-6]. TTR, 5(1), 265-271.

https://doi.org/10.7202/037116ar

Tous droits réservés (C) TTR: traduction, terminologie, rédaction — Les auteurs, 1992

Cecument est protégé par la loi sur le droit d'auteur. L'utilisation des services d'Érudit (y compris la reproduction) est assujettie à sa politique d'utilisation que vous pouvez consulter en ligne.

https://apropos.erudit.org/fr/usagers/politique-dutilisation/ 


\title{
La traduction, la terminologie et la rédaction en chronique (7)
}

\author{
Jean Delisle, Candace Séguinot, Claude \\ Tatilon, Sergio Viaggio, Marie-France Wagner
}

Lieven D'Hulst. Cent ans de théorie française de la traduction. De Batteux à Littré (1748-1847). Presses Universitaires de Lille, 1990, 256 p. ISBN 2-85939-370-6.

Dans la foulée du retour en force de l'histoire chez les traductologues, Lieven D'Hulst, professeur à l'Université d'Anvers, apporte une contribution importante et de qualité aux études sur la traduction abordées d'un point de vue historique. Cent ans de théorie française de la traduction se présente sous la forme d'un recueil d'une trentaine de textes theroriques et critiques couvrant la période qui s'étend de la fin de l'âge classique à la fin de l'époque romantique. Plus précisément, cet ouvrage fait ressortir la variété des techniques et des fonctions de la traduction entre 1748 et 1847 , tout en dégageant les enjeux de trois genres réflexifs: la théorie, la préface, le compte rendu.

A ces trois genres correspondent les trois grandes subdivisions de l'ouvrage: I - Parcours théoriques, II - Rhétorique des préfaces, III Stratégies de lecture. À l'intérieur de chacune de ces sections, introduites par une notice historique et descriptive bien structurée, les textes sont classés par ordre chronologique. Cet arrangement fait ressortir l'évolution de la pensée traductive tout au long des cent ans étudiés. Ainsi, la section des «Parcours théoriques» - onze extraits - s'ouvre par Charles Batteux (1748) et se termine par Émile Littré (1847). Entre ces deux pôles, on retrouve des auteurs-traducteurs réputés (D'Alembert, $\mathrm{M}^{\mathrm{m}}$ de Staël, Vigny) et d'autres moins connus pour qui n'est pas spécialiste de cette période 
(Beauzée, Vaultier, Loyson). Chaque traducteur fait l'objet d'une notice biobibliographique et d'un résumé de sa pensée sur l'art de traduire. Ces précieuses indications, si succinctes soient-elles, situent les traducteurs non seulement dans le temps, mais encore dans les courants littéraires ou théoriques de leur époque. Tout au long de l'ouvrage, enfin, l'auteur multiplie les notes explicatives de nature encyclopédique et érudite et, ce faisant, accentue le caractère didactique de son travail et en facilite la lecture. Comme il s'agit, en outre, d'un instrument de référence, une abondante bibliographie (pp. 237-248) et un index onomastique complètent utilement l'ouvrage.

Si les études et publications consacrées à l'histoire de la traduction au Moyen Âge et à la Renaissance abondent, ${ }^{1}$ plus rares et plus fragmentaires sont celles qui portent sur les $\mathrm{XVII}^{\mathrm{e}}$ et $\mathrm{XIX}^{\mathrm{e}}$ siècles. Cent ans de théorie française de la traduction vient presque combler un vide. Comme le fait observer l'auteur lui-même, la plupart des textes qui figurent dans son recueil «ne sont guère accessibles dans des éditions courantes», et l'Anthologie de la manière de traduire de Paul Horguelin (Linguatech, 1981) consacre tout au plus «une vingtaine d'extraits d'une page en moyenne à la période étudiée dans le présent volume» (p. 13, note 13). Le recueil de Lieven D'Hulst se distingue en outre de cette anthologie par la longueur des fragments reproduits: ceux-ci varient de trois à sept pages, alors que chez Paul Horguelin, ils dépassent rarement une page et demie. Il faut dire que ce dernier couvre une période de vingt siècles (de Cicéron à Jean Darbelnet).

La longueur des fragments a son importance, car si ceux-ci sont trop courts, il est difficile d'obtenir une idée fidèle de la manière de traduire d'un auteur. La démarche de Lieven D'Hulst «se distingue [donc] des recueils qui ont porté à la connaissance du public les «grands» témoins de la tradition occidentale anglaise, allemande, espagnole ou française, en ce qu'elle vise à procurer un instrument apte à mieux rendre compte du discours pris en lui-même et en relation avec la communication traductrice dans son ensemble» (p. 13). On peut affirmer que l'auteur a atteint son but. ( $J$ 'avoue cependant que la signification exacte du dernier membre de cette phrase m'échappe.)

1. Cf. notre compte rendu de l'ouvrage de Jeanette Beer, Medieval Translators and their Craft, (Meta, vol. XXXVI, $\mathrm{n}^{\infty}$ 2-3, juin-septembre 1991), pp. 511-514. 
Il convient de préciser que les textes de son recueil se rapportent tous à des traductions littéraires d'auteurs anciens et modernes. Au XVIII siècle, la réflexion sur la traduction appartient au domaine des BellesLettres, domaine «surtout départagé entre la critique et l'enseignement littéraires, qui relèvent de la rhétorique, et l'étude et l'enseignement des langues et du langage, domaine de la grammaire. La réflexion traductrice s'intéresse dès lors peu, jusqu'en plein XIX' siècle, aux textes scientifiques, à la philosophie, à l'histoire, ou aux genres semi-littéraires comme le récit de voyage» (p. 14).

Quelles impressions générales se dégagent de ce panorama de l'activité de traduction de 1748 à 1847 et de cette galerie imposante de traducteurs défendant des conceptions souvent diamétralement opposées de leur art? Des lecteurs cyniques pourraient s'interroger sur l'utilité de ce retour aux sources, au moment où la traductologie semble avoir quitté les ornières de l'empirisme artisanal et consolide ses bases scientifiques. Ces lecteurs souscriraient sûrement au jugement du linguiste Georges Mounin qui a écrit à propos de la masse des observations léguées par l'armée des traducteurs depuis Cicéron:

Il s'agit toujours de réflexions littéraires, qui constituent une masse énorme de témoignages plutôt que de recherches, une accumulation d'intuitions, des collections de recettes ou de généralités, voire de banalités répétées de siècle en siècle, ou encore de formulations catégoriques indémontrées, mêlées avec une richesse extraordinaire de faits concrets bien observés: un praticisme et un empirisme de bons artisans. ${ }^{2}$

A la lecture des fragments regroupés dans Cent ans de théorie française de la traduction, on est frappé de constater à quel point la réflexion sur la traduction à l'époque est encore enfermée dans des dichotomies, voire des apories: Faut-il traduire littéralement ou librement? Faut-il traduire les poètes anciens en vers ou en prose? Une traduction à la fois fidèle et élégante est-elle possible? Faut-il respecter la syntaxe du modèle original ou peut-on s'en écarter? Faut-il importer dans la traduction la couleur locale ou la gommer pour s'en tenir aux usages de la langue et de la culture réceptrices? Ce sont-là quelques exemples de questions que des générations

2. Linguistique et traduction (Bruxelles, Dessart et Mardaga, 1976), pp. 89-90. 
successives de traducteurs se sont posées inlassablement. Ces éternelles oppositions binaires mêlaient les préoccupations théoriques et méthodologiques, ce qui ne contribuait guère à clarifier le débat.

Toutes ces observations ont l'allure de «profession de foi», pour reprendre l'expression d'Henri Patin (p. 206), et n'ont rien de systématique, cela va sans dire. On force donc un peu le sens du mot «théorie», me semble-t-il, en l'appliquant aux «réflexions», aux «observations», aux «idées» sur la traduction exprimées par les traducteurs. Ceux-ci les formulaient, et Lieven D'Hulst l'a très bien démontré dans les textes de présentation de chacune des trois parties de son ouvrage, soit pour fonder la possibilité de la traduction et définir la notion de fidélité ( $«$ Parcours théoriques», p. 17ss), soit pour justifier leur «manière» ou aller au devant des reproches («Rhétorique des préfaces», p. 103ss), soit pour juger et critiquer des traductions dans des comptes rendus ( $\$$ Stratégies de lecture», pp. $170 \mathrm{ss})^{3}$. Il faut admettre, cependant, que le mot «théorie» se révèle bien utile et revêt le sens de «réflexion sur». Ce serait commettre une erreur, voire un anachronisme, que de lui donner le sens restrictif de «a series of statements, each of which is derived logically from a previous statement or from an axiom and which together have a strong power of explanation and prediction regarding a certain phenomenon", ${ }^{4}$ en l'occurrence le processus de la traduction et son résultat.

Le style métaphorique est une autre caractéristique de ces textes et nous confirme, si besoin était, que nous sommes bien dans le royaume des Belles-Lettres. Les images et les comparaisons pullulent pour décrire les réalités de la traduction. Certaines de ces images reviennent avec insistance. On ne compte plus les auteurs qui ont comparé une traduction avec une (mauvaise) copie d'un tableau. En outre, tout le cortège des anciennes catégories de la grammaire et de la rhétorique (tenues aujourd'hui comme

3. Dans une communication présentée à Belgrade en août 1990 , à l'occasion du XII Congrès mondial de la FIT, Lieven D'Hulst avait lui-même reconnu que «de tout temps, la pensée traductive a été encombrée de dichotomies, et a donc été tissée de répétitions».

4. James J. Holmes, «Translation Theory, Translation Studies, and the Translator», (la traduction une profession, Actes du VIII Congrès mondial de la FIT, publiés sous la direction de Paul Horguelin, Conseil des traducteurs et interprètes du Canada, 1978), p. 56. 
«objets d'antiquité» par la linguistique moderne) défilent sous nos yeux. Les expressions «génie de la langue», «force», «énergie», «harmonie», «grâce», «finesse», «coloris», «invention» se bousculent sous la plume des traducteurs décrivant leur art difficile, faute d'un «métalangage» précis pour en parler.

En revanche, les formules lapidaires et axiomes bien frappés abondent. Pour décrire les vertus du mot juste et de la concision, Charles Batteux écrit: «Quand les signes sont clairs, moins il y en a, plus ils sont vifs» (p. 30). «Les traducteurs sont dans le cas des voyageurs, dont les rapports ne sont pas toujours dignes de foi» (Bitaubé, p. 30). «Si le traducteur n'était interprète, il serait inutile» (Vigny, p. 94). «Les traductions sont pour un idiome ce que les voyages sont pour l'esprit» (Delille, p. 121). Toutes ces images, toute cette terminologie contribuent à recréer l'atmosphère de l'époque et font du livre de Lieven D'Hulst une lecture fort agréable pour qui s'intéresse le moindrement à l'histoire de la traduction.

Mais l'intérêt de Cent ans de théorie française de la traduction ne réside pas uniquement dans le rassemblement de textes épars par l'auteur. Il tient aussi au fait qu'il a su montrer, par le choix de ces textes et leur présentation, la grande vitalité de la démarche réflexive au cours de la période étudiée. Il fait clairement ressortir que la méthode d'un traducteur est historiquement conditionnée, que la tradition pèse de tout son poids sur sa manière d'aborder le modèle original, «manière» qui dépend aussi de son appartenance à une école littéraire ou de sa conception esthétique. Henri Meschonnic a d'ailleurs écrit à ce propos: «La notion de traduction est une notion historique. La traduction comme la pratique idéologique courante [..] se définit par le possible d'une époque. Le possible d'une époque est la somme de ses idées reçues». ${ }^{5}$ Cinquante ans avant Meschonnic, Paul Hazard avait tenu des propos similaires: «Tout traducteur subit, qu'il le veuille ou non, l'influence secrète du goût propre à sa nation; malgré lui, il fait passer dans sa version ses propres habitudes intellectuelles, ses façons de sentir [...] ${ }^{6}{ }^{6} C^{\prime}$ est ainsi qu'au fil des textes du recueil, on peut sentir l'évolution d'un genre, le passage progressif des «belles infidèles» aux traductions plus littérales et «colorées» du XIX ${ }^{e}$ siècle. Sous l'influence de

5. Pour la poétique II, (Paris, NRFGallimard, 1977), p. 321.

6. «L'invasion des littératures du Nord dans l'Italie du XVII' siècle», (La Revue de littérature comparée, t. I, 1921), p. 46. 
l'évolution sociologique, linguistique, culturelle et intellectuelle en France, les traducteurs ont manifesté le «désir d'ouvrir l'Étranger en tant qu'Étranger à [leur] propre espace de langage»?

Les traducteurs appartenant à l'ancien système (qui a coexisté pendant un certain temps, évidemment, avec le nouveau, embellissent leur modèle, ils «dorent l'or» et «parfument la rose» comme a si bien dit Henri Patin (p. 210) en paraphrasant deux vers de Shakespeare. A cette ancienne école appartiennent Jacques Amyot, Perrot d'Ablancourt, Hélène Dacier, l'abbé Prévost, qui tous «traduisaient au profit de la langue nationale» (Nisard, p. 216). «Dans le second système, poursuit Nisard, le traducteur devait tout simplement retourner la thèse, et sacrifier la langue nationale à l'auteur traduit. La traduction, disait ce système, n'a pas pour objet d'affubler un ancien du costume moderne, un latin de l'habit français, mais de forcer la langue qui traduit à se prêter à toutes les innovations, altérations, surcharges qui peuvent aider cet ancien, ce latin, à paraître dans sa véritable allure, avec son propre costume à lui [...]» (Ibid.) Si cette évolution a été possible, c'est, constate Chateaubriand, parce que «notre langue est devenue plus hardie et plus populaire» (p. 170). Les partisans de cette nouvelle façon de traduire ont nom, entre autres, Littré, Courier, Bignan, Chateaubriand, Cherbuliez, tous traducteurs de la première moitié du XIX siècle.

En nous offrant la possibilité de scruter les textes réflexifs et critiques sur la traduction, Lieven D'Hulst nous donne l'occasion de dépasser les affirmations à l'emporte-pièce comme celle de G. Mounin citée précédemment, et de multiplier les découvertes intéressantes, en particulier les diverses fonctions attribuées à la traduction. C'est ainsi qu'on se rend compte «qu'il n'y a pas de théorie unifiée de la traduction après 1750 , mais des théories qui se côtoient ou se concurrencent plus ou moins ouvertement [...]» (p. 18). Certaines d'entre elles (celle de Batteux, par exemple) sont largement tributaires de la grammaire et de la rhétorique auxquelles elles empruntent leurs concepts. Par ailleurs, selon $\mathbf{M}^{\mathrm{me}}$ de Staël, la traduction est un excellent remède contre l'appauvrissement des littératures nationales. Alfred de Vigny attribuait aussi à la traduction une fonction littéraire: traduire Shakespeare en français, c'est contribuer à renouveler le thêâtre

7. Antoine Berman, «La traduction et la lettre ou l'auberge de lointain», (Les Tours de Babel: Essais sur la traduction, Mauvezin, TransEurop-Repress, 1985), pp. 88-89. 
français. Pour leur part, l'abbé Jacques Delille et Étienne-Augustin de Wailly voient dans la pratique des traductions un instrument idéal d'enrichissement de la langue, un antidote contre la sclérose de l'expression.

En interrogeant les textes, il devient possible de connaître les devoirs que s'imposent les traducteurs au regard des qualités d'une bonne traduction. On découvre ainsi les multiples qualités dont doivent se parer les traductions, compte tenu des contraintes de tout ordre imposées par la culture adoptive. Vigny rappelle, non sans ironie, que les timides traducteurs français de Shakespeare ont mis 98 ans avant de se décider à dire tout haut sur scène un mouchoir!

En relisant ces anciens textes, on découvre que plusieurs notions contemporaines de la traductologie y sont présentes en germe. MarieClaude-Frédéric Vautier définit en 1812 les notions de «compensation» et d' «équivalence» d'une manière que ne récuseraient pas les comparatistes modernes. Beauzée, quant à lui, distingue habilement «version» et «traduction».

En somme, Cent ans de théorie française de la traduction fournit aux historiens de la traduction un riche matériau qu'ils sauront exploiter avec profit, je n'en doute pas. Cet ouvrage bien fait et présenté sous une fort jolie couverture, trouvera aussi une grande utilité en enseignement, puisque, comme il a déjà été dit, les études sur cette période sont peu nombreuses. «Le style de l'histoire, a écrit Charles Batteux, doit être clair, aisé, coulant et toujours soutenu» (p. 32). Telles sont les qualités de l'ouvrage de Lieven D'Hulst, qui a produit une cuvre utile et de haute tenue. Espérons qu'elle sera suivie de nombreuses autres.

Jean Delisle

Université d'Ottawa

\section{Wolfgang LÖRSCHER. Translation Performance, Translation Process, and Translation Strategies. A Psycho- linguistic Investigation. Tübingen, Gunter Narr, 1991. 307 p.}

Anyone interested in observational studies of translation will no doubt be familiar with the work of Wolfgang Lörscher through his recent articles on 